\title{
Medi \\ 60-jähriger Mann mit Husten und Dyspnoe nach Grippeschutzimpfung
} Quiz
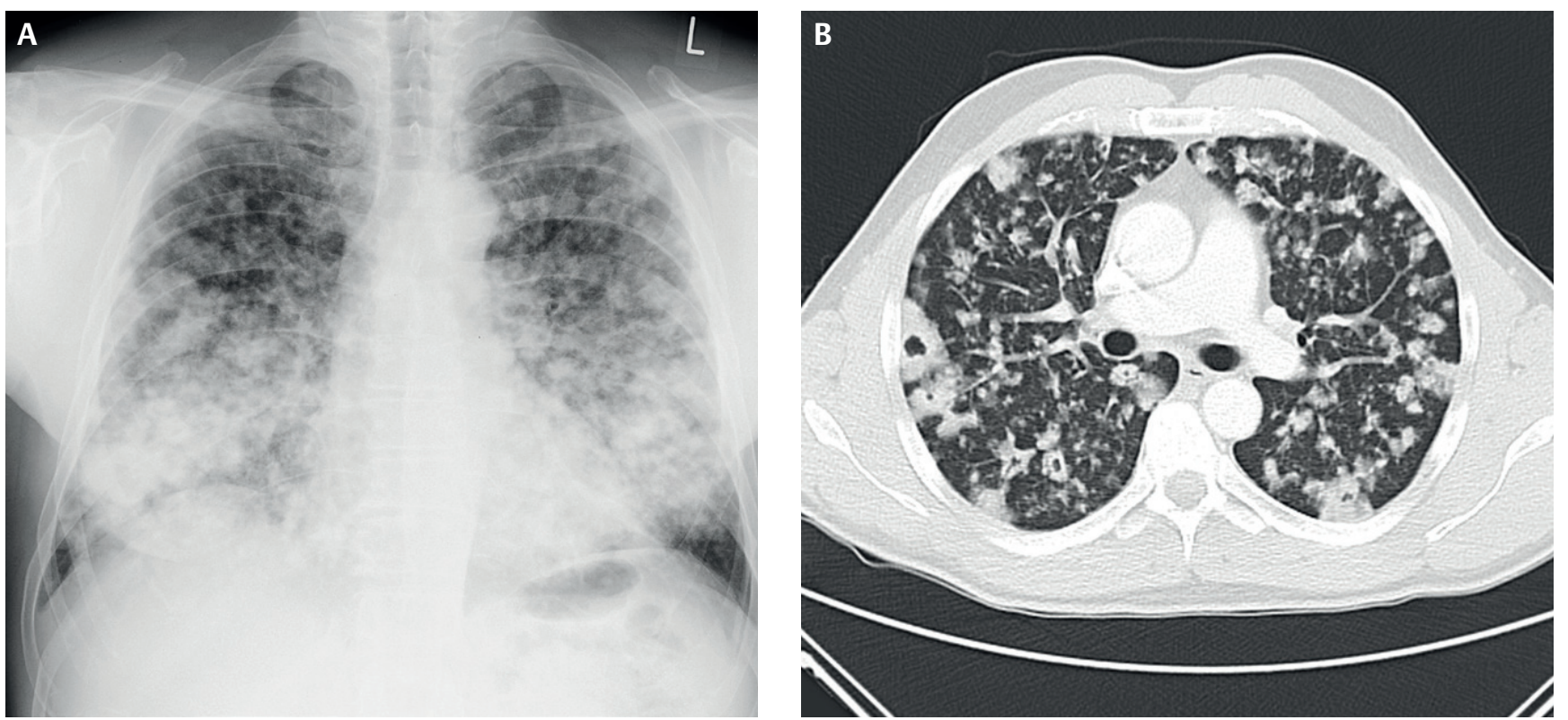

Abb. 1 Röntgen-Thorax (ap) (A), Thorax-CT (Lungenfenster, axiale Schnittführung) (B).

Dr. med. Leonie Kramer ${ }^{1}$

Kathrin Phiesel ${ }^{2}$

Prof. Dr. med. Holger Strunk ${ }^{1}$

${ }^{1}$ Radiologische Klinik, Universitätsklinikum Bonn

${ }^{2}$ Medizinische Klinik und Poliklinik I -

Allgemeine Innere Medizin,

Universitätsklinikum Bonn

Korrespondenz:

leonie.kramer@ukb.uni-bonn.de
Einen Monat nach einer saisonalen Influenzaschutzimpfung entwickelt sich bei einem 60-jährigen Mann ein produktiver Husten. Innerhalb von 3 Monaten kommen Heiserkeit und eine zunehmende Dyspnoe hinzu. Verschiedene Antibiotika lindern die Beschwerden nicht. Eine Bronchoskopie ergibt eine leichtgradige, nicht-putride Bronchitits. Die bronchoalveoläre Lavage und die Laborparameter sind unauffällig. Im Sputum finden sich zudem keine säurefesten Stäbchen. Eine Röntgenaufnahme und eine CT des Thorax zeigen zwei pathologische Befunde ( $\mathbf{A b b} \mathbf{1}$ A, B).

- Welche Befunde sind es?

- Erlauben die Befunde in Kombination mit der Klinik eine Verdachtsdiagnose?

- Wenn ja, welche?

- Sind Differenzialdiagnosen möglich?

- Wenn ja, welche?

Auflösung Seite 1547

Der Beitrag wurde gemäß folgendem Erratum korrigiert:

Die Fallzahlen der Mediquize aus Heft 19 und 20 wurden falsch fortgeführt. Korrekt muss es heißen: „37-jährige Schwangere mit Luftnot und wechselnden EKG-Veränderungen“ (Dtsch Med Wochenschr 2015; 140: 1423) ist Fall 3184, und „60-jähriger Mann mit Husten und Dyspnoe nach Grippeschutzimpfung“ (Dtsch Med Wochenschr 2015; 140: 1537) ist Fall 3185.
Dtsch Med Wochenschr 2015; 140: 1537

(c) Georg Thieme Verlag KG · Stuttgart · New York · ISSN 0012-0472 


\title{
Medi Quiz
}

\section{0-jähriger Mann mit Husten und Dyspnoe nach Grippeschutzimpfung}

\author{
Quiz siehe S. 1537
}
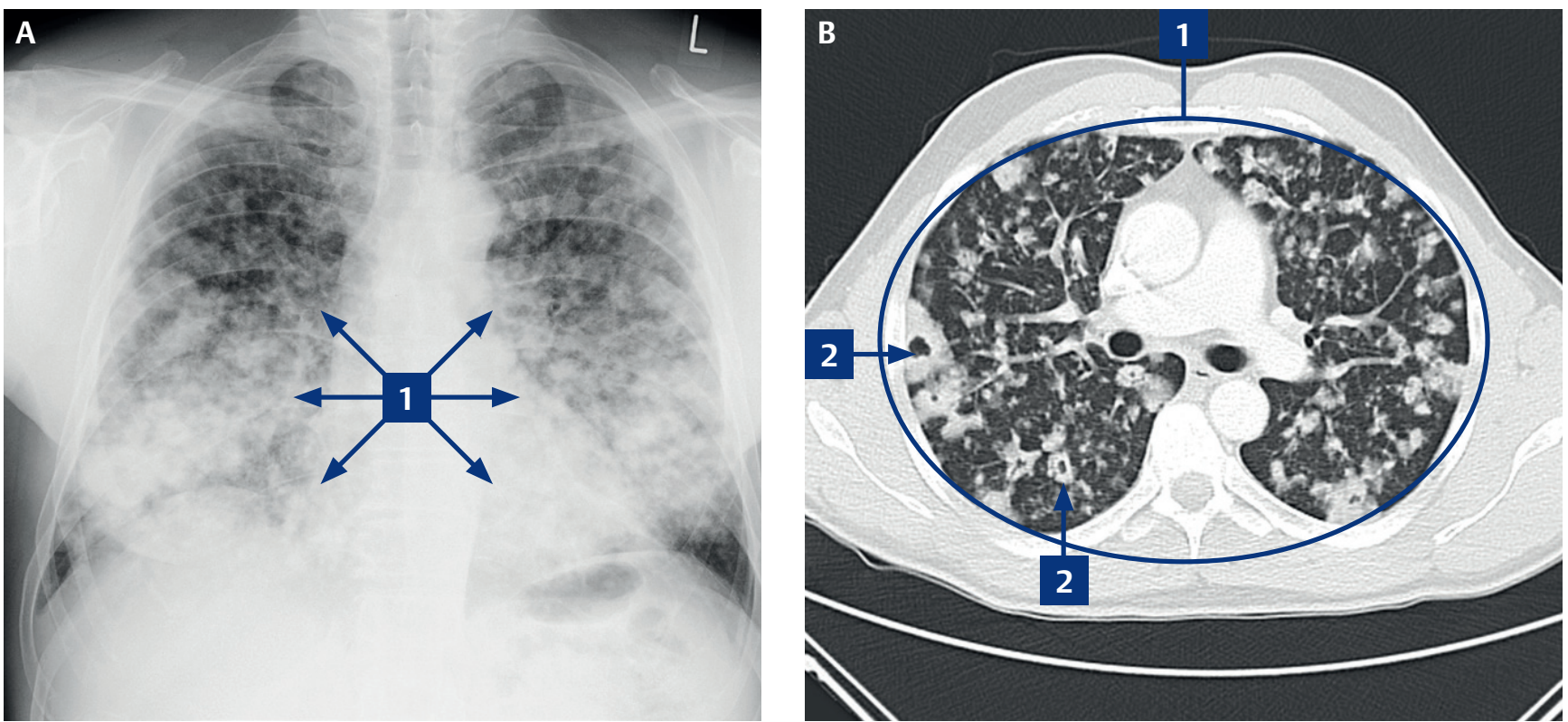

\section{Befunde}

1. disseminierte, konfluierende, noduläre Verdichtungen mit

2. zentral lufthaltigen Arealen (Pseudokavitationen)

\section{Verdachtsdiagnosen}

- septische Embolien

- Malignome (primär oder Metastasen)

\section{Differenzialdiagnosen}

- entzündliche Infiltrate (z.B. atypische Pneumonie, Tuberkulose)

- immunologische Veränderungen (z.B. Wegener'sche Granulomatose)

\section{Erläuterung}

Aufgrund der computertomografischen Veränderungen bei gleichzeitig nur milder Klinik waren septische Embolien oder ein malignes Geschehen die primären Verdachtsdiagnosen. Gegen entzündliche und immunologische Prozesse sprachen die klinischen und anamnestischen Angaben sowie die unauffälligen laborchemischen Infektparameter. Um die Diagnose zu sichern, wurde die rechte Lunge CT-gesteuert punktiert (nach befundloser Bronchoskopie). Die Histologie ergab ein hoch differenziertes muzinöses Adenokarzinom der Lunge. Im Anschluss wurde eine palliative Chemotherapie mit Cisplatin, Pemetrexed und Avastin begonnen.

Das Adenokarzinom der Lunge gehört zur Gruppe der NSCLC („non-small-cell-lungcancer"). Nach der neuen IASLC/ATS/ERSKlassifikation aus dem Jahr 2011 werden pulmonale Adenokarzinome eingeteilt in - präinasive Läsionen,

- minimalinvasive Adenokarzinome,

- invasive Adenokarzinome und

- Varianten des invasiven Adenokarzinoms.

Das invasiv muzinöse Adenokarzinom gehört zur letztgenannten Gruppe [1, 3]. Da diese Tumoren häufig Kontakt zum
Bronchialstamm haben, metastasieren sie oft auf bronchoaerogenem Weg [1]. Dies ist im geschilderten Fallbericht bildmorphologisch gut nachvollziehbar. Die beschriebene Tumorentität ist mit einer schlechten Prognose verbunden [2]. Sie wird oft erst in fortgeschrittenen Stadien diagnostiziert.

Daher sollte auch bei primär unauffälliger Bronchoskopie und fehlenden Infektparametern ein muzinöses Adenokarzinom differenzialdiagnostisch abgeklärt werden. Hierzu kann eine frühzeitige, CT-gesteuerte Biopsie erwogen werden.

\section{Literatur}

1 Petersen I. Adenokarzinome der Lunge die neue Klassifikation, Zentralbl Chir 2013; 138: 16-24

2 Watanabe $\mathrm{H}$, Saito $\mathrm{H}$, Yokose $\mathrm{T}$ et al. Relation between thin-section computed tomography and clinical findings of mucinous adenocarcinoma. Ann Thorac Surg 2015; 99: 975-981

3 Petersen I. Morphologische und molekulare Diagnostik des Lungenkarzinoms. Dtsch Arztebl 2011; 108: 523-531 